\section{P121 Nutrition-Related Recommendations and Dietary Habits Following Diabetes Diagnosis: Findings From the 2015 Kenya STEPS-Survey}

Kilee Kimmel, BS, kekilee@bsu.edu, Ball State University, 2000 W University Ave, Muncie, IN 47306; Teresia Mbogori, PhD, Ball State University

Background: Health care in low- and middle-income countries continues to focus on reducing under-nutrition despite the increasing prevalence of overweight, obesity, and non-communicable diseases (NCDs). Diabetes Mellitus (DM) is an emerging NCD in Kenya, however research on nutrition-related recommendations and behaviors following diagnosis is limited.

Objective: To identify nutrition-related recommendations provided by Health Care Professionals (HCP) to DM patients, and identify dietary behaviors following diagnosis.

Study Design, Settings, Participants: A cross-sectional study was conducted among 4,500 Kenyan adults (18-69 years) participating in the 2015 Kenya STEPwise approach to surveillance study (STEPS) sponsored by the World Health Organization (WHO).

Measurable Outcome/Analysis: Participants' eight hours fasting blood glucose (FBG) values were measured. The following variables were created using FBG values to define DM status according to ADA recommendations: normal $=<5.6 \mathrm{mmol} / \mathrm{l} \quad(<100 \mathrm{mg} / \mathrm{dL}) ;$ prediabetes $=5.6$ $6.9 \mathrm{mmol} / \mathrm{l}(100-125 \mathrm{mg} / \mathrm{dL}) ;$ and $\mathrm{DM}=\geq 7.0 \mathrm{mmol} / \mathrm{l}(\geq 126$ $\mathrm{mg} / \mathrm{dL})$. Descriptive statistics and cross tabulations were used to determine current DM status based on FBG provided, previous DM diagnosis by HCP, dietary recommendations provided after diagnosis, and current dietary patterns followed by the participants.

Results: $10.2 \%$ and $2.4 \%$ of the participants presented with FBG values indicative of prediabetes and DM respectively. Fourty-four percent of the individuals presenting with DM reported that they had not received a formal diagnosis by a HCP. $40 \%, 43 \%$, and $76 \%$ of participants who had received a formal diagnosis stated that they had not been advised to limit intake of refined sugar, eat five servings of fruit/vegetables a day, and reduce alcohol consumption respectively. Although $75 \%$ of those diagnosed with DM believed lowering sugar in the diet was 'very important', about $28 \%$ reported that they did not limit sugary drink consumption and $14 \%$ consumed processed foods high in sugar at most meals, or every week.

Conclusion: Despite the increasing DM prevalence and diagnosis in Kenya, many remain undiagnosed. Health care improvements warrant the need for increased prevention methods and dietary guidance following diagnosis.

Funding: None.

\section{P122 The Effects of a Campus Dining Tour Intervention on First Year Students Perception of Healthfulness of Environment}

Paige Pappadackis, BS, South Dakota State University; Kendra Kattelmann, PhD, RDN, FAND, LN, kendra. kattelmann@sdstate.edu, South Dakota State University,
Box 2275A, SWG 425, Brookings, SD 57007; Lee Weidauer, PhD, ATC, South Dakota State University; Lacey McCormack, PhD, MPH, RD, LN, EP-C, South Dakota State University; Sarah Colby, PhD, RD, University of Tennessee

Background: Many young adults experience unwanted weight gain upon entering college. Making healthy choices in a food environment with a plethora of convenience and fast foods is important for preventing unwanted weight gain.

Objective: To determine if a Healthy Campus Dining Tour intervention improves perception, behavior, and priorities related to healthier choices on campus

Study Design, Settings, Participants: Participants were recruited for this quasi-experimental study from freshman introductory classes at a land-grant public university and assigned to intervention or a control group. Intervention participants completed a 50-minute Healthy Campus Dining Tour that educated on how to make healthier choices at each of the campus dining locations (vending, convenience, kiosk, and all-you-can-eat dining). Measureable Outcome/Analysis: Both groups were assessed pre- and post-intervention for agreement with questions assessing perception of healthful food choices in the campus environment (12 questions), frequency of certain healthful dietary behaviors (12 questions) and importance of food choice priorities (24 questions). Outcomes were dichotomized as more or less positive responses and logistic regression was used to determine odds of responses between intervention and control.

Results: One hundred and twenty participants $(n=45$ intervention, $\mathrm{n}=75$ control) that completed the surveys. Most participants were 18 years of age, female, white, freshmen status, and lived in a campus residence hall. Greater odds of perceiving healthier foods in restaurants [OR(CI), 2.9 (1.3-6.5)], dining halls [2.1, (0.9-4.7)], and vending [8.1 (3.4-18.7)] were seen in intervention group versus control group. Odds of increased frequency of healthful behaviors and increased importance of food choice priorities did not differ between groups.

Conclusion: Participation in the Healthy Campus Dining Tour intervention was associated with greater odds of agreeable perceptions regarding availability of healthy foods on campus. Additional programming and environmental changes may be necessary to change food behavior and priorities.

Funding: None.

\section{Behavior and Education Theory}

\section{P123 A Qualitative Investigation into Nutrition Literacy Assessment in Outpatient Clinics}

Nicholas Marchello, MS, RD,LD, nmarchello@kumc.edu, University of Kansas Medical Center, 3901 Rainbow Blvd, Kansas City, KS 66160; Christine Daley, PhD, MA, SM, University of Kansas Medical Center; Breta Alstrom, BS, RD, LD, University of Kansas Medical Center; Cora Burgoyne, 


\section{P123 (continued)}

$B S$, University of Kansas Medical Center; Heather Gibbs, $P h D, R D$, University of Kansas Medical Center

Background: Nutrition literacy is a unique subset of health literacy, a leading predictor of poor health outcomes. Understanding a patient's nutrition literacy may help dietitians provide interventions tailored to a patient's nutrition knowledge weaknesses. While tools exist to evaluate nutrition literacy, it is unclear whether dietitians currently assess patients for nutrition literacy.

Objective: To examine nutrition literacy assessment among practicing outpatient dietitians, barriers to implementing a nutrition literacy survey into clinical practice, and possible solutions to these barriers.

Study Design, Settings, Participants: Semi-structured interviews, divided into four sampling frames, with dietitians $(\mathrm{n}=28)$ from multiple outpatient settings were conducted across two Midwestern states. Similar interviews were conducted with outpatient clinic managers $(n=7)$, providing a wider viewpoint.

Measurable Outcome/Analysis: Questions addressed clinic work flow, assessing nutrition literacy, and barriers to implementing a survey into practice. All interviews were transcribed, coded, and analyzed by trained study personnel.

Results: All dietitians stated they use no nutrition literacy assessment tools in clinical practice; subjective assessments are used. Barriers to survey implementation included time to complete the survey $(79 \% / \mathrm{n}=22$ of interviewees), logistics in administering the survey $(75 \% / \mathrm{n}=21)$, patient motivation $(75 \% / \mathrm{n}=21)$, literacy levels of patients $(54 \% / \mathrm{n}=15)$, and clinic staff support $(32 / \mathrm{n}=9)$. Clinic managers stated barriers to survey implementation included patient motivation $(86 \% /$ $\mathrm{n}=6)$, devoting staff to a non-revenue generating task $(71 \% / \mathrm{n}=5)$, logistics in administering the survey $(71 \% /$ $\mathrm{n}=5)$, and time to complete the survey $(57 \% / \mathrm{n}=4)$. Solutions include survey administration prior to the patient's appointment ( $79 \%$ of dietitians $/ \mathrm{n}=22,86 \%$ of managers $/ n=6)$, motivating patients to complete the survey prior to their visit $(29 \% / \mathrm{n}=8,100 \% / \mathrm{n}=7)$, and reducing survey length $(21 \% / \mathrm{n}=6,43 \% / \mathrm{n}=3)$.

Conclusion: While multiple barriers to evaluating nutrition literacy in clinical settings exist, potential solutions to these barriers exist. Further research on the implementation of nutrition literacy assessment in the outpatient clinic setting is needed.

Funding: KU Medical Center Research Institute.

\section{P124 A Qualitative Study of the Attitudes and Practices of Hispanic Mothers of Young Children Around Feeding Practices and Food Choices}

CynthiaLebron, MPH, clebron@miami.edu, University of Miami Miller School of Medicine, 1120 NW 14 St, Miami, FL 33136; Tamisha Guzman, BS, Florida International University; Yaray Agosto, MPH, Florida International University; Danielle Sutton, MPH, Florida International University; Mark Stoutenberg, PhD, MPH, University of
Chattanooga; Sarah Messiah, PhD, MPH, UT Southwestern; Sara M. StGeorge, PhD, University of Miami

Background: There are socioeconomic factors particular to Hispanic populations that influence the way parents feed their children. As obesity continues to be a public health issue with substantial inequities across race and ethnicity, it is critical to understand Hispanic parents' food choices and feeding practices.

Objective: To identify the behavioral, environmental, and cognitive factors that influence food choices and feeding behaviors of Hispanic mothers of children ages 05 years.

Study Design, Settings, Participants: Snowball sampling was used to recruit participants. Participants $(n=30)$ were included in the study if they were over 18 , and selfidentified as Hispanic/Latina and a mother of a child between the ages of 0-5 years. Semi-structured interviews were conducted in either English or Spanish and were audio recorded and transcribed verbatim.

Measurable Outcome/Analysis: Each interview consisted of a brief survey and open-ended questions based on Social Cognitive Theory (SCT) constructs. The questions centered on pediatric guidelines for healthy eating. A thematic analysis using a combined deductive and inductive approach is currently being used to analyze transcriptions.

Results: Emerging themes include challenges, attitudes, and strategies for both breastfeeding and introducing solid foods. Mothers had especially strong attitudes towards breastfeeding and expressed the importance of it but conversely described the multitudes of problems they experienced. Mothers described issues feeding their older children due to taste preferences and the methods they used to encourage eating, including bribing or tricking the child by hiding nutritious foods in recipes. Participants also described numerous sources of influence that shaped their feeding choices, the most common being other moms, healthcare providers, their culture, and the internet.

Conclusion: Hispanic mothers are actively seeking information from many different sources that impact how they feed their young children. Understanding their trusted sources and how it influences the SCT constructs is an important step in preventing early childhood obesity.

Funding: None.

\section{P125 Assessment of Active Learning Techniques and Experiential Learning Coursework on Dietetic Student's Perceptions of Learning}

Karen Gordon,PhD, RD, LD, klowry@kent.edu, Kent State University, School of Health Sciences, 1225 Theatre Dr, Kent, OH 44242

Objective: To assess the role of experiential/service learning and active student learning approaches on students' perceptions of the experiences and approaches.

Use of Theory or Research: As accreditation standards become more competency-based, it is critical for educators to understand how students may meet these competencies

Continued on page 589 